\title{
On Lie rings of torsion groups
}

\author{
Consuelo Martínez ${ }^{1}\left(\right.$ D - Efim Zelmanov ${ }^{2,3}$
}

Received: 16 April 2016/ Accepted: 23 May 2016 / Published online: 10 June 2016

(C) The Author(s) 2016. This article is published with open access at Springerlink.com

\begin{abstract}
We prove that the Lie ring associated to the lower central series of a finitely generated residually-p torsion group is graded nil.
\end{abstract}

Keywords Torsion group $\cdot$ Nil Lie algebra

Mathematics Subject Classification 20F40 - 20F45 $\cdot 20$ F50

\section{Introduction}

Let $G$ be a group. A descending sequence of normal subgroups $G=G_{1}>G_{2}>\cdots$ is called a central series if $\left[G_{i}, G_{j}\right] \subseteq G_{i+j}$ for all $i, j \geq 1$. The direct sum of abelian groups $L(G)=\oplus_{i \geq 1} G_{i} / G_{i+1}$ is a graded Lie ring with Lie bracket $\left[a_{i} G_{i+1}, b_{j} G_{j+1}\right]=\left[a_{i}, b_{j}\right] G_{i+j+1} ; a_{i} \in G_{i}, b_{j} \in G_{j}$.

Of particular interest are the lower central series: $G_{1}=G, G_{i+1}=\left[G_{i}, G\right], i \geq 1$, and, for a fixed prime number $p$, the Zassenhaus series (see $[7,8]$ ).

Communicated by S. K. Jain.

The first author has been partially supported by MTM 2013-45588-C3-1-P and GRUPIN 14-142 and the second one by the NSF.

Consuelo Martínez

cmartinez@uniovi.es

1 Departamento de Matemáticas, Universidad de Oviedo, C/ Calvo Sotelo s/n, 33007 Oviedo, Spain

2 Department of Mathematics, UCSD, La Jolla, CA 92093-0112, USA

3 KAU, Jeddah, Saudi Arabia 
Let $p$ be a prime number. We say that a group $G$ is residually- $p$ if the intersection of all normal subgroups of indices $p^{i}, i \geq 1$, is trivial.

A graded Lie ring $L=L_{1}+L_{2}+\cdots$ is called graded nil if for an arbitrary homogeneous element $a \in L_{i}$ the adjoint operator $a d(a): x \rightarrow[a, x]$ is nilpotent.

The main instrument in the study of the Burnside problem in the class of residually $p$-groups is the connection between torsion in the group $G$ and graded nilness in the Lie algebra $L(G)$ of the Zassenhaus series (see [6-9]).

In this paper we prove this connection for the lower central series and for an arbitrary central series of $G$.

Theorem 1 Let $\Gamma$ be a finitely generated residually-p torsion group. Let $\Gamma=\Gamma_{1}>$ $\Gamma_{2}>\cdots$ be the lower central series of $\Gamma$. Then the Lie ring $L(\Gamma)=\oplus_{i \geq 1} \Gamma_{i} / \Gamma_{i+1}$ is graded nil.

Note that the known important classes of torsion groups: Golod-Shafarevich groups (see [2,3]), Grigorchuck groups [4] and Gupta-Sidki groups [5] are residually- $p$.

We say that a (possibly infinite) group $\Gamma$ is a $p$-group if for an arbitrary element $g \in \Gamma$ there exists $k \geq 1$ such that $g p^{k}=1$. Clearly, for a residually $p$-group being torsion and being a $p$-group are equivalent.

Theorem 2 Let $\Gamma$ be a p-group. Let $\Gamma=\Gamma_{1}>\Gamma_{2}>\cdots$ be a central series. Then the Lie ring $L(\Gamma)=\oplus_{i \geq 1} \Gamma_{i} / \Gamma_{i+1}$ is locally graded nil.

In other words, we claim that an arbitrary finitely generated graded subalgebra of $L(\Gamma)$ is graded nil.

\section{Definitions and results}

Let $\Gamma$ be a residually- $p$ group and let $G$ be its pro- $p$ completion (see [1]). For an element $y \in G$ let $\left\langle y^{G}\right\rangle$ denote the closed normal subgroup of $G$ generated by $y$. Let $\left[y^{G}, y^{G}\right]$ denote the closed commutator subgroup of $\left\langle y^{G}\right\rangle$.

For elements $g_{1}, g_{2}, \ldots, g_{n} \in G$ let $\left[g_{1}, g_{2}, \ldots, g_{n}\right]=\left[g_{1},\left[g_{2},\left[\ldots, g_{n}\right]\right] \ldots\right]$ be their left-normed commutator.

We will need the following equalities which can be found in [1]:

(1) For an arbitrary integer $k \geq 1$ we have $[y, x]^{k}=\left[y^{k}, x\right] \bmod \left[y^{G}, y^{G}\right]$;

(2) $\left[y, x^{k}\right]=[y, x]^{\left(\begin{array}{c}k \\ 1\end{array}\right)}[y, x, x]^{\left(\begin{array}{c}k \\ 2\end{array}\right)} \ldots[y, \underbrace{x, \ldots x}_{k}]^{\left(\begin{array}{c}k \\ k\end{array}\right)} \bmod \left[y^{G}, y^{G}\right]$.

Let $p$ be a prime number. The equalities (1) and (2) imply that

(3) $c=\left[y, x^{p}\right][y, \underbrace{x, \ldots, x}_{p}]^{-1}=\left[y^{\left(\begin{array}{c}p \\ 1\end{array}\right)}, x\right]\left[y^{\left(\begin{array}{c}p \\ 2\end{array}\right)}, x, x\right] \ldots[y^{\left(\begin{array}{c}p \\ p-1\end{array}\right)}, \underbrace{x, \ldots x}_{p-1}] \bmod$ $\left[y^{G}, y^{G}\right]$.

Hence $\left[y^{p}, x\right]=c\left[\left[y^{p}, x\right], x\right]^{-\left(\begin{array}{c}p \\ 2\end{array}\right) / p} \ldots[\left[y^{p}, x\right], \underbrace{x, \ldots x}_{p-2}]^{-\left(\begin{array}{c}p \\ p-1\end{array}\right) / p} \bmod \left[y^{G}, y^{G}\right]$.

Iterating this process and the use of equalities (1) and (2) we conclude that there exists an infinite sequence of nonnegative integers $k_{i} \geq 0$ such that 
(4) $\left[y^{p}, x\right]=c[c, x]^{k_{1}}[c, x, x]^{k_{2}} \ldots \bmod \left[y^{G}, y^{G}\right]$.

Let $\rho$ be a left-normed group commutator, $\rho=\left[g_{1}, \ldots g_{m}\right]$, where each element $g_{i}$ is either equal to y or to $x^{p^{k}}$ for some $k \geq 1$. Let $d_{x}(\rho)$ denote the sum of powers of $x$ involved in $\rho$ and $d_{y}(\rho)$ the number of times the element $y$ occurs in $\rho$.

Lemma 1 Let $\rho=\left[y, x^{p^{i_{1}}}, x^{p^{i_{2}}}, \ldots, x^{p^{i_{k}}}\right], d_{y}(\rho)=1,0 \leq i_{1}, \ldots, i_{k} \leq l-1$. Then $\rho$ is a converging product of commutators $\sigma$ of types:

(i) $\sigma=\left[y, x^{p^{j_{1}}}, x^{p^{j_{2}}}, \ldots, x^{p^{j_{s}}}\right]$, where $d_{y}(\sigma)=1$, no more than one integer of $j_{1}, \ldots, j_{s}$ is different from 0 and $d_{x}(\sigma) \geq d_{x}(\rho)$

(ii) $\sigma=\left[y, x^{p^{j_{1}}}, \ldots, y, \ldots\right]$, where $d_{y}(\sigma) \geq 2$ and $\left(d_{y}(\sigma)-1\right) p^{l}+d_{x}(\sigma) \geq d_{x}(\rho)$.

Proof Suppose that $i_{\alpha}, i_{\beta} \geq 1,1 \leq \alpha \neq \beta \leq k$. We will represent $\rho$ as a product of commutators of types (i) and (ii) and of commutators of type

(iii) $\rho^{\prime}=\left[y, x^{p^{j_{1}}}, x^{p^{j_{2}}}, \ldots, x^{p^{j_{s}}}\right]$, where $0 \leq j_{1}, \ldots, j_{s} \leq l-1$ and $0 \leq d_{x}\left(\rho^{\prime}\right)>$ $d_{x}(\rho)$

and type

(iv) $\rho^{\prime \prime}=\left[y, x^{p^{j_{1}}}, x^{p^{j_{2}}}, \ldots, x^{p^{j_{s}}}\right]$, where $0 \leq j_{1}, \ldots, j_{s} \leq l-1$ and $d_{x}\left(\rho^{\prime \prime}\right)=d_{x}(\rho)$, $s>k$.

Iterating we will get rid of commutators (iii) and (iv).

Without loss of generality we will assume that $\alpha=1, \beta=2$ and $1 \leq i_{1} \leq i_{2}$.

By (3) we have

$\left[y, x^{p^{i_{1}}}\right]=\left[y^{\left(\begin{array}{l}p \\ 1\end{array}\right)}, x^{p^{i_{1}-1}}\right] \ldots[y^{\left(\begin{array}{c}p \\ p-1\end{array}\right)}, \underbrace{x^{p^{i_{1}-1}}, \ldots, x^{p^{i_{1}-1}}}_{p-1}][y, \underbrace{x^{p^{i_{1}-1}}, \ldots, x^{p^{i_{1}-1}}}_{p}]$

$\bmod \left[y^{G}, y^{G}\right]$.

Now $\rho$ is a product of commutators of the form

$[y^{\left(\begin{array}{l}p \\ t\end{array}\right)}, \underbrace{x^{p^{i_{1}-1}}, \ldots, x^{p^{i_{1}-1}}}_{t}, x^{p^{i_{2}}}, \ldots, x^{p^{i_{k}}}], 1 \leq t \leq p-1$; of the commutator

$[y, \underbrace{x^{p^{i_{1}-1}}, \ldots, x^{p^{i_{1}-1}}}_{p}, x^{p^{i_{2}}}, \ldots, x^{p^{i_{k}}}]$ and of commutators that involve at least two occurrences of $y$ and the powers $x^{p^{i_{1}-1}}, x p^{i_{2}}, \ldots, x p^{i_{k}}$ (see [7]).

The latter commutators are commutators of type (ii). The commutator $[y, \underbrace{x^{p^{i_{1}-1}}, \ldots, x^{p^{i_{1}-1}}}_{p}, x^{p^{i_{2}}}, \ldots, x^{p^{i_{k}}}]$ satisfies condition (iv). Hence it remains to con-

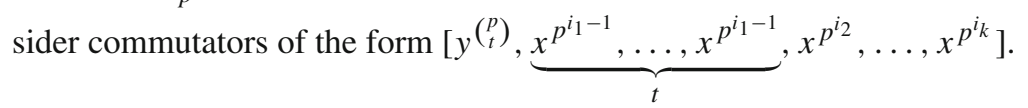

Since $p \mid\left(\begin{array}{l}p \\ t\end{array}\right), 1 \leq t \leq p-1$, we will consider the commutator

$$
[y^{p}, \underbrace{x^{p^{i_{1}-1}}, \ldots, x^{p^{i_{1}-1}}}_{t}, x^{p^{i_{2}}}, \ldots, x^{p^{i_{k}}}] .
$$


Modulo longer commutators we can move the power $x^{p^{i_{2}}}$ to the left. By (4) we get

$$
\left[y^{p}, x^{p^{i_{2}}}\right]=\sigma[\sigma, x]^{s_{1}}[\sigma, x, x]^{s_{2}} \ldots
$$

where $\sigma=\left[y, x^{p^{i_{2}+1}}\right][y, \underbrace{x^{p^{i_{2}}}, \ldots, x^{p_{2}}}_{p}]^{-1} \bmod \left[y^{G}, y^{G}\right]$.

The commutators $[y, x^{p^{i_{2}+1}}, \underbrace{x^{p^{i_{1}-1}}, \ldots, x^{p^{i_{1}-1}}}_{t}, \ldots x^{p^{i_{k}}}]$ and

$[y, \underbrace{x^{p^{i_{2}}}, \ldots, x^{p^{i_{2}}}}_{p}, \underbrace{x^{p^{i_{1}-1}}, \ldots, x^{p^{i_{1}-1}}}_{t}, \ldots x^{p^{i_{k}}}]$ are of type (iii) since $i_{2} \geq i_{1}$ and therefore $p^{i_{2}+1}+p^{i_{1}-1}>p^{i_{2}}+p^{i_{1}}$.

This finishes the proof of the lemma.

Consider again the commutator $\rho=\left[y, x^{p^{i_{1}}}, \ldots, x^{p^{i_{k}}}\right]$. Suppose that $x^{p^{l}}=1$. Consider the $l$-tuple $\operatorname{ind}(\rho)=\left(k_{l-1}, \ldots, k_{0}\right), k_{i} \in Z_{\geq 0}$, where $k_{i}$ is the number of times $i$ occurs among $i_{1}, \ldots, i_{k}$. Clearly, $k_{0}+k_{1}+\cdots k_{l-1}=k$.

Consider the length-lex order in $Z^{l} \geq 0:\left(\alpha_{1}, \ldots, \alpha_{l}\right)>\left(\beta_{1}, \ldots, \beta_{l}\right)$ if either $\sum \alpha_{i}>\sum \beta_{i}$ or $\sum \alpha_{i}=\sum \beta_{i}$ and $\left(\alpha_{1}, \ldots, \alpha_{l}\right)>\left(\beta_{1}, \ldots, \beta_{l}\right)$ lexicographically.

Lemma 2 Let $x, y \in G, x^{p^{l}}=1, y^{p^{s}}=1$. A commutator $\rho=\left[y, x^{p_{1}^{i_{1}}}, \ldots, x^{p^{i_{k}}}\right]$ such that $d_{x}(\rho) \geq(s+1) p^{l}$ can be represented as a product of commutators $\sigma=$ $\left[y, x^{p^{j_{1}}}, \ldots, y, \ldots, x^{p^{j q}}\right]$, where $d_{y}(\sigma) \geq 2$ and $\left(d_{y}(\sigma)-1\right) p^{l}+d_{x}(\sigma) \geq d_{x}(\rho)$.

Proof We will show that $\rho$ is a (converging) product of commutators of the form $\sigma_{1}$ and $\sigma_{2}$, where $d_{y}\left(\sigma_{1}\right) \geq 2,\left(d_{y}\left(\sigma_{1}\right)-1\right) p^{l}+d_{x}\left(\sigma_{1}\right) \geq d_{x}(\rho)$ for commutators of the form $\sigma_{1}$, whereas commutators of the form $\sigma_{2}$ look as $\sigma_{2}=\left[y, x^{p^{j_{1}}}, \ldots, x^{p^{j t}}\right]$ with $d_{x}\left(\sigma_{2}\right)>d_{x}(\rho)$ or $d_{x}\left(\sigma_{2}\right)=d_{x}(\rho)$ and $i n d\left(\sigma_{2}\right)>\operatorname{ind}(\rho)$. Then, applying this assertion to commutators of the form $\sigma_{2}$ and iterating we will get rid of commutators $\sigma_{2}$.

We claim that at least one $i, 0 \leq i \leq l-1$, occurs in $i_{1}, \ldots, i_{k}$ not less than $p$ times. Indeed, otherwise $d_{x}(\rho) \leq(p-1)\left(1+p+\cdots+p^{l-1}\right)$, which contradicts our assumption that $d_{x}(\rho) \geq(s+1) p^{l}$.

Suppose that $i$ occurs in $i_{1}, \ldots, i_{k}$ not less than $\mathrm{p}$ times and $i$ is the smallest in $\left\{i_{1}, \ldots, i_{k}\right\}$ with this property. Moving the occurrences of $i$ to the left, modulo longer commutators, we assume $i_{1}=\cdots=i_{p}=i$.

By (2) we have

$$
[y, \underbrace{x^{p^{i}}, \ldots, x^{p^{i}}}_{p}]=\left[y, x^{p^{i+1}}\right]\left[y^{\left(\begin{array}{c}
p \\
1
\end{array}\right)}, x^{p^{i}}\right]^{-1} \ldots[y^{\left(\begin{array}{c}
p \\
p-1
\end{array}\right)}, \underbrace{x^{p^{i}}, \ldots, x^{p^{i}}}_{p-1}]^{-1} \tau_{1} \ldots \tau_{q},
$$

where $\tau_{j}$ are commutators that involve $y$ at least twice.

The commutator $\sigma^{\prime}=\left[y, x^{p^{i+1}}, x^{p^{i} p+1}, \ldots, x^{p^{i k}}\right]$ has greater index than $\rho$. Indeed, $d_{x}\left(\sigma^{\prime}\right)=d_{x}(\rho)$, but $i n d\left(\sigma^{\prime}\right)$ is lexicographically greater than ind $(\rho)$. 
For a commutator $\tau_{j}^{\prime}=\left[\tau_{j}, x^{p^{i} p+1}, \ldots, x^{p^{i_{k}}}\right]$, we have

$$
d_{x}\left(\tau_{j}^{\prime}\right) \geq d_{x}(\rho)-(p-1) p^{i}
$$

Hence, $p^{l}\left(d_{y}\left(\tau_{j}^{\prime}\right)-1\right)+d_{x}\left(\tau_{j}^{\prime}\right) \geq p^{l}+d_{x}(\rho)-(p-1) p^{i}>d_{x}(\rho)$.

Consider now the commutator $\rho^{\prime}=\left[y^{p}, x^{p^{i}}, x^{p^{i_{p}+1}}, \ldots, x^{p^{i k}}\right]$.

We claim that there exists $j \in\left\{i_{p+1}, \ldots, i_{k}\right\}$ such that $j \geq i$. Indeed, otherwise all integers in $\left\{i_{p+1}, \ldots, i_{k}\right\}$ are smaller than $i$ and therefore occur $\leq(p-1)$ times. Hence,

$d_{x}(\rho) \leq p p^{i}+(p-1)\left(1+p+\cdots+p^{i-1}\right)=p^{i+1}+p^{i}-1<2 p^{l} \leq(s+1) p^{l}$,

which contradicts the assumption of the lemma.

Moving $x p^{j}$ to the right end in $\rho^{\prime}$ modulo longer commutators we will assume that $i_{k}=j \geq i$.

Consider the commutator $\rho^{\prime \prime}=\left[y^{p}, x^{p^{i}}, x^{p^{i} p+1}, \ldots, x^{p^{i_{k+1}}}\right]$. We have $d_{x}\left(\rho^{\prime \prime}\right)=$ $d_{x}(\rho)-(p-1) p^{i}-p^{j} \geq s p^{l}$. By the induction assumption on $s$ the commutator $\rho^{\prime \prime}$ is a product of commutators $w$ in $y^{p}$ and $x$, each commutator involves $\mu=\mu(w) \geq 2$ elements $y^{p}$ and $(\mu-1) p^{l}+d_{x}(w) \geq d_{x}\left(\rho^{\prime \prime}\right)$. We will assume that $w=\left[w_{1}, \ldots, w_{\mu}\right]$, $w_{j}=\left[y^{p}, \ldots\right], 1 \leq j \leq \mu$.

Remark Any commutator that has degree $\geq \mu+1$ in $y$ and degree $\geq d_{x}(w)$ in $x$ fits the requirements of the lemma since $\mu p^{l}+d_{x}(w) \geq d_{x}\left(\rho^{\prime \prime}\right)+p^{l} \geq d_{x}(\rho)$.

The commutator $\left[w_{1}, \ldots, w_{\mu}, x^{p^{j}}\right]$ is equal to a product

$$
\left[\left[w_{1}, x^{p^{j}}\right], w_{2}, \ldots, w_{\mu}\right]\left[w_{1},\left[w_{2}, x^{p^{j}}\right], \ldots\right] \ldots\left[w_{1}, \ldots,\left[w_{\mu}, x^{p^{j}}\right]\right]
$$

modulo longer commutators (see the Remark above).

Consider $\left[w_{1}, \ldots,\left[w_{\nu}, x^{p^{j}}\right], \ldots, w_{\mu}\right]$.

In $\left[w_{\nu}, x^{p^{j}}\right]$ move $x^{p^{j}}$ to the left position next to $y^{p}$ modulo longer commutators (see the Remark above).

By (4), $\left[y^{p}, x^{p^{j}}\right]=c[c, x]^{k_{1}}[c, x, x]^{k_{2}} \ldots \tau_{1} \ldots \tau_{q}$, where $c=\left[y, x^{p^{j+1}}\right][y, \underbrace{x^{p^{j}}, \ldots, x^{p^{j}}}_{p}]^{-1} ; \tau_{1}, \ldots \tau_{q} \in\left[y^{G}, y^{G}\right] ; d_{x}\left(\tau_{1}\right), \ldots, d_{x}\left(\tau_{q}\right) \geq p^{j}$.

If the commutator $\left[y^{p}, x^{p^{j}}\right]$ is replaced by one of $\tau_{1}, \ldots, \tau_{q}$ then see the Remark.

If $\left[y^{p}, x^{p^{j}}\right]$ is replaced by $c$ then

$$
\begin{aligned}
& d_{x}\left(\left[w_{1}, \ldots, w_{\nu-1}, c, w_{\nu+1}, \ldots, w_{\mu}\right]\right) \geq \\
& \quad d_{x}\left(\left[w_{1}, \ldots, w_{\mu}, x^{p^{j}}\right]\right)+(p-1) p^{j} d_{x}(w)+p j+1 .
\end{aligned}
$$

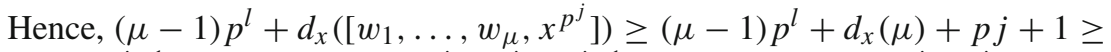
$d_{x}\left(\rho^{\prime \prime}\right)+p^{j+1}=d_{x}(\rho)-(p-1) p^{i}-p^{j}+p^{j+1}=d_{x}(\rho)+(p-1)\left(p^{j}-p^{i}\right) \geq d_{x}(\rho)$. 
Since $d_{y}\left(\left[w_{1}, \ldots, w_{\mu}, x^{p^{j}}\right]\right) \geq 2$, this commutator satisfies the requirements of the lemma. If the commutators $\left[y^{p}, x^{p^{j}}\right]$ is replaced by $[c, \underbrace{x, \ldots, x}_{t}]^{k_{t}}$, then $(\mu-$ 1) $p^{l}+d_{x}\left(\left[w_{1}, \ldots, w_{\nu-1},[c, \underbrace{x, \ldots, x}_{t}]^{k_{1}}, w_{\nu+1}, \ldots, w_{\mu}\right]\right)>d_{x}(\rho)$.

This finishes the proof of the lemma.

Lemma 3 Let $x \in G_{i}, x^{p^{l}}=1, y \in G_{j}, y^{p^{s}}=1$. Suppose that $j \geq 2 i p^{l}$. Then $\left(y G_{j+1}\right) \operatorname{ad}\left(x G_{i+1}\right)^{(s+1) p^{l}}=0$ in the Lie algebra $L=\sum_{k=1}^{\infty} G_{k} / G_{k+1}$.

Proof By Lemma 2 the group commutator $\rho=[y, \underbrace{x, \ldots, x}]$ can be represented as a $(s+1) p^{l}$

product of commutators $w=\left[w_{1}, \ldots, w_{\mu}\right], \mu \geq 2$, where each $w_{k}$ is a commutator of the type $w_{k}=\left[y, x^{p^{j_{1}}}, \ldots, x^{p^{j_{r}}}\right],(\mu-1) p^{l}+d_{x}(w) \geq d_{x}(\rho)=(s+1) p^{l}$.

By Lemma 1 each $w_{k}$ is a product of commutators of type (i) or (ii). A commutator of type (ii) just increases the degree in $y$. Let $\left[y, x^{p^{j_{1}}}, \ldots, x^{p^{j_{r}}}\right]$ be a commutator of type (i). So all $j_{1}, \ldots, j_{r}$, except possibly one, are equal to 0 . This implies that

$$
\left[y, x^{p^{j_{1}}}, \ldots, x^{p^{j_{r}}}\right] \in G_{j+i\left(p^{j_{1}}+\cdots+p^{j_{r}}-\left(p^{l-1}-1\right)\right)} .
$$

Hence, $w \in G_{d}$, where $d=\mu j+i d_{x}(w)-\mu i\left(p^{l-1}-1\right) \geq j+(\mu-1) i p^{l}+(\mu-$ 1) $i p^{l}+i d_{x}(w)-\mu i\left(p^{l-1}-1\right) \geq j+i d_{x}(\rho)+i\left[(\mu-1) p^{l}-\mu\left(p^{l-1}-1\right)\right]$.

Now it remains to notice that $(\mu-1) p^{l}-\mu\left(p^{l-1}-1\right)>0$. We showed that $d>j+i d_{x}(\rho)$, which implies the lemma.

Lemma 4 The Lie ring $L(\Gamma)$ is weakly graded nil, i.e., for arbitrary homogeneous elements $a, b \in L(\Gamma)$ there exists $n(a, b) \geq 1$ such that bad $(a)^{n(a, b)}=0$.

Proof Let $a \in \Gamma_{i}, a^{p^{l}}=1$. Let $n(a)=2 i p^{l}$. By Lemma 3, for an arbitrary element $b \in \Gamma_{j}, j \geq n(a)$, there exists an integer $n(a, b) \geq 1$ such that $[b, \underbrace{a, a, \ldots, a}_{n(a, b)}] \in$ $G_{j+i n(a, b)+1}$.

Since $\Gamma$ is a torsion group it follows that for an arbitrary $k \geq 1$ the subgroup $\Gamma_{k}$ has finite index in $\Gamma$, hence $\Gamma_{k}$ is open in $\Gamma$. The subgroup $G_{k}$ is the completion of $\Gamma_{k}$. Hence $\Gamma \cap G_{k}=\Gamma_{k}$.

We proved that $\operatorname{bad}(a)^{n(a, b)}=0$ in $L(\Gamma)$. Now let $b$ be an arbitrary homogeneous element from $L(\Gamma)$. Then the degree of the element $b^{\prime}=\operatorname{bad}(a)^{n(a)}$ is greater than $n(a)$. Hence, $\operatorname{bad}(a)^{n(a)+n\left(a, b^{\prime}\right)}=b^{\prime} a d(a)^{n\left(a, b^{\prime}\right)}=0$, which finishes the proof of the lemma.

Lemma 5 Let L be a Lie algebra over a field Z/pZ generated by elements $x_{1}, \ldots, x_{m}$. Let $a \in L$ be an element such that $x_{i} a d(a)^{p^{k}}=0,1 \leq i \leq m$. Then Lad $(a)^{p^{k}}=(0)$.

Proof The algebra $L$ is embeddable in its universal associative enveloping algebra $U(L)$. Let $a^{p^{k}}$ be the power of the element $a$ in $U(L)$. For an arbitrary element $b \in L$ we have $\operatorname{bad}(a)^{p^{k}}=\left[b, a^{p^{k}}\right]$. If the element $a^{p^{k}}$ commutes with all generators $x_{1}, \ldots, x_{m}$ then $\left[L, a^{p^{k}}\right]=\operatorname{Lad}(a)^{p^{k}}=(0)$, which finishes the proof of the lemma. 
Lemma 6 Let $L$ be a Lie ring generated by elements $x_{1}, \ldots, x_{m}$. Suppose that $p^{l} L=$ (0). Let $a \in L$ be an element such that $x_{i}$ ad $(a)^{p^{k}}=0,1 \leq i \leq m$. Then $\operatorname{Lad}(a)^{p^{k} l}=$ (0).

Proof By Lemma 5 we have $\operatorname{Lad}(a)^{p^{k}} \subseteq p L$. Hence $L\left(a d(a)^{p^{k}}\right)^{l} \subseteq p^{l} L=(0)$, which proves the lemma.

Proof of Theorem 1 Let $x_{1}, \ldots, x_{m}$ be generators of the group $\Gamma$. Then the elements $x_{i} \Gamma_{2}, 1 \leq i \leq m$, generate the Lie ring $L(\Gamma)$. Let $p^{l}$ be the maximum of orders of the elements $x_{1}, \ldots, x_{m}$, so $x_{i}^{p^{l}}=1,1 \leq i \leq m$. Then $p^{l}\left(x_{i} \Gamma_{2}\right)=0$ in the Lie ring $L(\Gamma)$. Hence $p^{l} L(\Gamma)=(0)$.

Let $a$ be a homogeneous element of $L(\Gamma)$. By Lemma 4 there exists $k \geq 1$ such that $\left(x_{i} \Gamma_{2}\right) a d(a)^{p^{k}}=0$ for $i=1, \ldots, m$. Now Lemma 6 implies that $L(\Gamma) a d(a)^{p^{k} \cdot l}=$ $(0)$, which finishes the proof of Theorem 1 .

Proof of Theorem 2 Without loss of generality we assume that $\cap_{i} \Gamma_{i}=(1)$. We view the subgroups $\left\{\Gamma_{i} \mid i \geq 1\right\}$ as a basis of neighborhoods of 1 thus making $\Gamma$ a topological group. Let $G$ be a completion of $\Gamma$ in this topology. Let $G_{i}$ be the closure of $\Gamma_{i}$ in $G$. Then $G_{i} \cap \Gamma=\Gamma_{i}$ and $G=G_{1}>G_{2}>\cdots$ is a central series of the group $G$. Arguing as in Lemmas 3, 4 we conclude that the Lie ring $L(\Gamma)=\bigoplus_{i \geq 1} \Gamma_{i} / \Gamma_{i+1}$ is weakly graded nil. Choose homogeneous elements $a_{1}, \ldots, a_{m} \in L(\Gamma)$. Since $\Gamma$ is a $p$-group it follows that there exists $l \geq 1$ such that $p^{l} a_{i}=0,1 \leq i \leq m$. Consider the subring $L^{\prime}$ of $L(\Gamma)$ generated by $a_{1}, \ldots, a_{m}, p^{l} L^{\prime}=(0)$. If $a$ is a homogeneous element from $L^{\prime}$ and $a_{i} a d(a)^{p^{k}}=0,1 \leq i \leq m$, then by Lemma 6 we have $L^{\prime} a d(a)^{p^{k} \cdot l}=(0)$, which finishes the proof of Theorem 2.

Open Access This article is distributed under the terms of the Creative Commons Attribution 4.0 International License (http://creativecommons.org/licenses/by/4.0/), which permits unrestricted use, distribution, and reproduction in any medium, provided you give appropriate credit to the original author(s) and the source, provide a link to the Creative Commons license, and indicate if changes were made.

\section{References}

1. Dixon, J.M., du Sautoy, M.P.F., Mann, A., Segal, D.: Analytic pro-p groups, London Math. Soc. Lecture Notes Series, vol. 157. Cambridge University Press, Cambridge (1991)

2. Ershov, M.: Golod-Shafarevich groups: a survey. Int. J. Algebra Comput. 22, Article ID 1230001 (2012). doi:10.1142/S0218196712300010

3. Golod, E.S.: On nil algebras and residually finite p-groups. Izv. Akad. Nauk SSSR Ser. Mat. 28, 273-276 (1964)

4. Grigorchuk, R.I.: Degrees of growth of finitely generated groups and the theory of invariant means. Izv. Akad. Nauk SSSR Ser. Mat. 48, 939-985 (1984)

5. Gupta, N., Sidki, N.: On the Burnside problem for periodic groups. Math. Z. 182, 385-386 (1983)

6. Higman, G.: Lie ring methods in the theory of finite nilpotent groups. In: Proc. Internat. Congress Math. 1958, pp. 307-312. Cambridge Univ. Press, New York (1960)

7. Vaughan-Lee, M.: The restricted Burnside problem, 2nd edn. London Mathematical Society Monographs, New Series 8. The Clarendon Press. Oxford University Presss, New York (1993)

8. Zelmanov, E.: Nil rings and periodic groups, KMS Lecture Notes in Mathematics. Korean Mathematical Society, Seoul (1992)

9. Zelmanov, E.: Lie ring methods in the theory of nilpotent groups, Groups 93 Galway/St. Andrews, v.2, London Math. Soc. Lecture Note Ser., vol. 212, pp. 567-585. Cambridge Univ. Press, Cambridge (1995) 